Background Cutaneous lupus erythematosus (CLE) is an often disfiguring autoimmune disease affecting systemic lupus erythematosus (SLE) patients and a roughly similar number of CLEonly patients without systemic disease. Effective therapies for CLE are limited, and additional studies are needed to better understand the cellular composition and mediators of CLE pathology in order to improve treatment modalities.

Methods Lesional and non-lesional skin biopsies and peripheral blood mononuclear cells (PBMCs) from 7 patients with active CLE lesions and 14 healthy control skin biopsies were subjected to scRNA-seq on the $10 \mathrm{X}$ platform. Cells were clustered using Seurat and annotated according to putative cell type-specific markers. Keratinocytes (KCs), fibroblasts (FBs), T cells, and myeloid cells were sub-clustered and cell subsets annotated. Cytokine signatures were generated by treating KCs and FBs with panels of cytokines and identifying induced genes. For $\mathrm{KC}$ and $\mathrm{FB}$ sub-clustering analyses, each cell was scored for each cytokine signature. Cell-cell communication was examined using CellPhoneDB to perform ligand-receptor analysis for cell types. To further investigate cellular interactions, imaging mass cytometry and spatial transcriptomics were performed, and cell signatures derived from scRNA-seq were used to plot the proximity of different cell types in discoid lupus lesions. Finally, myeloid cells were identified from skin and PBMC samples and subclustered. Pseudotime analysis was performed on cells bridging the connection between circulating and skin-resident myeloid cells.

Results Both lesional and non-lesional SLE/CLE KCs exhibited strong type I interferon scores. Lesional and nonlesional SLE/ CLE skin exhibited dramatic shifts in cell-cell crosstalk; CD16 + DCs were highly enriched in CLE lesions relative to control and were among the most active communicators as expressers of both ligands and receptors. Spatial transcriptomics demonstrated CD16+ DCs localizing most prominently in the superficial dermis, enabling interaction with KCs. This was confirmed using imaging mass cytometry. Pseudotime analysis of paired circulating and skin myeloid cells revealed CD16+ DCs may arise from non-classical monocytes, with discrete shifts in myeloid cell transcriptional states, including a robust IFN education in the skin, detectable across this transition.

Conclusions Non-lesional skin of patients with SLE and CLE exists in a type I IFN-rich, 'prelesional' state. This affects gene transcription in all major skin cell types and dramatically alters cell-cell communication. Non-classical monocytes may infiltrate this environment to become CD16+ DCs that engage in crosstalk with diverse cell types as one of the earliest steps in the evolution of CLE.

\section{THE ROLE OF NEUTROPHILS IN THE CLINICAL SEVERITY OF LUPUS NEPHRITIS PATIENTS WITH CONCURRENT SKIN DISEASE}

Lais Osmani, Jason Pettus, Alecia Roy, Sladjana Skopelja-Gardner*. Dartmouth Hitchcock Medical Center, Lebanon NH, USA

10.1136/lupus-2021-lupus21century.8

Background Skin disease affects $>80 \%$ of lupus patients and has been linked to lupus nephritis (LN) flares. We recently found that skin inflammation caused by ultraviolet light leads to increased expression of renal inflammatory and injury markers as well as proteinuria, mediated by neutrophils, which migrated from the inflamed skin to the kidneys. These data prompted the hypothesis that presence of concurrent skin disease in lupus patients leads to worse nephritis mediated by neutrophils.

Methods Lupus nephritis biopsies $(n=43)$ were retrieved from the Dartmouth Hitchcock Medical Center tissue bank and grouped: (i) LN with concurrent skin rash (malar, subacute, or discoid $\mathrm{n}=15)$ and (ii) $\mathrm{LN}$ without any rash $(\mathrm{n}=6)$ at the time of LN diagnosis (class IV). Patients with inconclusive or incomplete skin findings were excluded. The study included only female patients. Clinical and laboratory data (absolute neutrophil (ANC) and lymphocyte (ALC) counts, serum creatinine, glomerular filtration rate (GFR), autoantibody titers, and complement) were collected by chart review. Histologic and immunofluorescence data were extracted from biopsy reports. Student's t-test was performed to detect statistical significance between patient groups.

Results Presence of skin rash at the time of LN flare was indicative of more active disease, reflected by higher ANA, antidsDNA IgG, and low C3 and C4 levels. LN patients with concurrent skin disease had a higher neutrophil count $(\mathrm{p}<$ $0.05)$ and neutrophil-lymphocyte ratio $(\mathrm{p}<0.01)$, but not ALC, compared to $\mathrm{LN}$ patients without a concurrent skin rash. High neutrophil counts in the presence of skin disease associated with lower GFR $(<60 ; \mathrm{p}<0.01)$. This association was not seen in the absence of concurrent skin disease and rash alone did not predict GFR. Analysis of kidney immunofluorescence revealed that concurrent skin disease at the time of LN flare associated with greater IgA deposition. Increased renal IgA levels associated with higher neutrophil but not lymphocyte counts $(\mathrm{p}<0.01)$. This was specific to IgA as no associations with IgG or IgM deposition were detected.

Conclusions Our study provides novel findings that suggest neutrophils may be the pathogenic link between skin inflammation and LN: (i) higher neutrophil counts are found in the presence of a skin rash at the time of LN flare, (ii) neutrophilia but not lymphocytosis in the presence of a skin rash associates with worse kidney function, and (iii) high neutrophils in the presence of skin rash associate with increased renal IgA. These data provide a novel model of IgA-driven activation of neutrophils leading to kidney injury initiated by skin inflammation.

\section{LYMPHATIC DYSFUNCTION IN LUPUS PHOTOSENSITIVITY}

\begin{abstract}
1,2William G Ambler*, 2,3 Noa Schwartz, ${ }^{4}$ Jin Yeon Shin, ${ }^{4}$ Rahgu Kataru, ${ }^{2}$ Camila Carballo, ${ }^{2,5}$ Scott Rodeo, ${ }^{4}$ Babak Mehrara, ${ }^{1,2,6}$ Theresa T Lu. 'Division of Pediatric Rheumatology, Department of Rheumatology, Hospital for Special Surgery, New York, NY, USA; ${ }^{2}$ HSS Research Institute, Hospital for Special Surgery, New York, NY, USA; ${ }^{3}$ Department of Medicine, Division of Rheumatology, Albert Einstein College of Medicine, Bronx, NY, USA; ${ }^{4}$ Department of Surgery, Division of Plastic and Reconstructive Surgery, Memorial Sloan Kettering Cancer Center, New York, NY, USA; ${ }^{5}$ Department of Orthopedics, Hospital for Special Surgery, New York, NY, USA; ${ }^{6}$ Department of Microbiology and Immunology, Weill Cornell Medicine, New York, NY, USA
\end{abstract}

\subsection{6/lupus-2021-lupus21century.9}

Background The lymphatic system is composed of vessels which carry fluid, soluble molecules, and cells from peripheral tissue to draining lymph nodes. Photosensitivity, an exaggerated inflammatory response in response to ultraviolet radiation (UVR), is present in most patients with Systemic Lupus Erythematosus (SLE). Lymphatic dysfunction has been shown to induce photosensitivity in wild-type models, thus we 
hypothesized that lymphatic dysfunction could contribute to photosensitivity in SLE.

Methods We examined MRL/lpr lupus prone mice for lymphatic function by injecting Evan's Blue into the ear and measuring retention. Ear thickness and flow cytometric analysis were used to assess photosensitivity. Lymphatic drainage was manipulated using two approaches. First, we used manual lymphatic drainage (MLD) in the MRL/lpr mice. MLD improved lymphatic drainage and reduced photosensitivity. Second, we induced a lupus phenotype in a novel mouse model with enhanced lymphatic function (inducible lymphatic endothelial cell specific PTEN KO) using topical imiquimod.

Results MRL/lpr mice had greater Evan's blue retention compared to controls suggesting lupus prone mice have impaired lymphatic drainage. MLD improved lymphatic drainage and reduced photosensitivity. PTEN KO mice had reduced photosensitivity and reduced systemic immune activation.

Conclusions This data suggests that lymphatic dysfunction contributes to photosensitivity in murine lupus and improving lymphatic flow, even with simple MLD, can ameliorate photosensitivity. Future studies will determine the etiology of lymphatic dysfunction in murine lupus and the mechanism of lessened photosensitivity with improved lymphatic drainage. If similar immune circuitry defects are present in patients with SLE, altering lymphatics could be a novel target for new therapeutics.

Acknowledgments Lupus Research Alliance

\section{0 - Transcriptomics}

\section{DIFFERENTIALLY EXPRESSED TRANSCRIPTS ASSOCIATED WITH LUPUS RISK LOCI IDENTIFY PATHOGENIC DISEASE PATHWAYS}

Mikhail Olferiev*, Dina Greenman, Jeffrey Zhang-Sun, David Fernandez, Kyriakos A Kirou, Mary K Crow. Hospital for Special Surgery, New York, USA

10.1136/lupus-2021-lupus21 century. 10

Background Genome-wide association studies (GWAS) have identified single nucleotide polymorphisms (SNPs) that tag genomic sites with a high statistical association with a diagnosis of SLE. As most risk SNPs mark regulatory rather than coding regions of the genome, the functional impact of the risk loci on molecular mechanisms and pathways has not been clearly defined. In addition, how disease-associated loci differentially impact pathogenic mechanisms in females and males with SLE has not been explored. We analyzed RNA sequencing data relevant to previously identified risk loci to understand the molecular pathways impacted by genetic variation.

Methods Forty subjects (10 SLE females; 10 SLE males; 10 healthy females; 10 healthy males) were identified for study, with careful matching of SLE and healthy donor subjects for age and ethnicity. Next generation RNA sequencing was performed and unsupervised clustering of transcript expression displayed for genes previously identified in GWAS as near SNPs associated with a diagnosis of SLE. Identified pathways were also investigated using an expanded RNAseq dataset, including 30 patients with lupus nephritis, 29 patients with non-renal SLE and 3 healthy donors.

Results Four major clusters of gene transcripts were identified, along with multiple subclusters. The most striking transcript subcluster preferentially expressed in lupus males identified mechanisms involved in nucleic acid sensing and type I interferon production (IRF7, IFIH1, IKBKE, TLR7, JAK2, CXorf21/TASL, and SLC15A4). Another subcluster that favored males included $\mathrm{T}$ and $\mathrm{B}$ cell transcripts associated with generation of autoantibody producing B cells (ITGAX, IRF5, SH2B3, TET3, NOTCH4, ICAM4). The cluster with transcripts showing decreased expression in SLE included DEF6 and PHRF1, potentially contributing to impaired transcriptional regulation of lymphocyte function in patients. Genes located in the MHC (ATF6B, NOTCH4, MICB, TNXB, ITPR3) or an $\mathrm{X}$ chromosome risk locus (IRAK1, TMEM187, MECP2, NAA10, HCFC1) were distributed among different transcript subclusters, suggesting the broad impact of risk haplotypes at those loci on regulation of multiple disease-associated pathways. Of interest is the observation that PHRF1 and IRF7, adjacent genes identified by a risk SNP (rs4963128), distributed to distinct clusters, with PHRF1 decreased and IRF7 increased in SLE patients. The expanded SLE dataset was also analyzed to further characterize lupus risk-associated functional transcript clusters.

Conclusion Genes located near lupus risk SNPs are differentially expressed in patients with SLE and cluster based on functional relationships. Transcripts differentially expressed in male patients suggest important involvement of nucleic acid sensing pathways in their disease.

\section{TRANSCRIPTOMIC ANALYSIS REVEALS A CRITICAL REGULATORY ROLE FOR CD8 T CELLS IN A MOUSE MODEL OF SYSTEMIC LUPUS ERYTHEMATOSUS}

${ }^{1}$ Andrea R Daamen ${ }^{*},{ }^{2}$ Chih-Hao Chang, ${ }^{2}$ Derry C Roopenian, ${ }^{1}$ Amrie C Grammer, ${ }^{1}$ Peter E Lipsky. 'AMPEL BioSolutions and the RILITE Research Institute, Charlottesville, VA, USA; ${ }^{2}$ The Jackson Laboratory, Bar Harbor, ME, USA

\subsection{6/lupus-2021-lupus21century. 1}

Background Pre-clinical mouse models are invaluable tools to investigate the mechanisms driving systemic lupus erythematosus (SLE) and identify therapeutic targets to treat human disease. The BXSB.Yaa (Yaa) mouse spontaneously develops SLE-like disease, that is accelerated by the loss of CD8 T cells in BXSB.Yaa.CD8 $\mathrm{a}^{-/-} \mathrm{IL} 15^{-/-}$(DKO) mice, suggesting that CD8 $\mathrm{T}$ cells have a protective role against autoimmunity in this model. The role of CD8 T cells in human SLE remains unclear as studies have found conflicting associations with increased disease severity or with a loss of regulatory activity. Therefore, we carried out transcriptome analysis of Yaa and DKO mice to clarify the role of CD8 T cells in SLE.

Methods Six week old 'pre-disease' mice were examined to determine the impact of CD8 T cells before the development of inflammatory disease. Gene expression profiles from spleens of 6 week old BXSB.B6 (CTL), Yaa, and DKO mice were obtained by RNA-seq. $\log _{2}$ gene expression values were normalized between datasets using housekeeping genes. Differential enrichment of immune cell and pathway gene modules curated for analysis of lupus mouse datasets was determined by Gene Set Variation Analysis (GSVA). Immune cell population GSVA scores were then correlated with functional pathways by linear regression.

Results Spleens from 6 week BXSB.Yaa mice showed evidence of extensive immune activation. GSVA revealed shared enrichment of SLE-associated lymphocyte populations including germinal center (GC) B cells and T follicular helper (Tfh) cells in 